\title{
Die Neoklassische Ökonomik und der Romantische Konsumismus: Ideologische Bremsklötze einer "Großen Transformation" zur Nachhaltigkeit
}

\section{Uwe Krüger \& Juliane Pfeiffer}

Keywords: Ideologiekritik, mentale Infrastrukturen, Kapitalismus, Lehrbuchökonomie, Volkswirtschaftslehre, Neoklassik, Romantik, Werbung, Journalismus

\section{Abstract}

Ausgehend von der Forderung einer "Großen Transformation“ der Weltgesellschaft hin zu einer nachhaltigen Lebens- und Wirtschaftsweise wird hier gefragt, mit welchen mentalen Infrastrukturen und Ideologien die Transformationsresistenz insbesondere westlich-kapitalistischer Gesellschaften erklärt werden kann. Wir schlagen das neoklassische Paradigma der Wirtschaftswissenschaft („Lehrbuchökonomie“) vor, das auf der Produktionsseite für Nachhaltigkeitsfragen blind macht, und den Romantischen Konsumismus, der Konsumptionssteigerungen in der breiten Bevölkerung begünstigt. Beide Überzeugungssysteme werden beschrieben und kritisiert, anschließend werden diesbezügliche Aufgaben einer transformativen Kommunikationswissenschaft skizziert.

Krüger, Uwe, und Juliane Pfeiffer. 2019. „Die Neoklassische Ökonomik und der Romantische Konsumismus: Ideologische Bremsklötze einer "Großen Transformation" zur Nachhaltigkeit". In Ideologie, Kritik, Öffentlichkeit. Verhandlungen des Netzwerks Kritische Kommunikationswissenschaft, herausgegeben von Uwe Krüger und Sebastian Sevignani, 200-225. Universität Leipzig. DOI: 10.36730/ideologiekritik.2019.10

Wir danken Mandy Tröger, Paul Lehmann, Sebastian Sevignani und Hendrik Theine für ihre konstruktive Kritik und wertvollen Hinweise zum Manuskript.

Uwe Krüger | Universität Leipzig | uwe.krueger@uni-leipzig.de Juliane Pfeiffer | Universität Leipzig | juliane.pfeiffer@uni-leipzig.de 
„I don't care who writes a nation's laws - or crafts its advanced treaties - if I can write its economics textbooks."

(Paul A. Samuelson, Wirtschaftsnobelpreisträger und Autor des einflussreichen Lehrbuchs Economics)

\section{Die "Große Transformation" und das Wachstum}

Ausgangspunkt unserer Überlegungen ist die Diagnose des Wissenschaftlichen Beirats der Bundesregierung Globale Umweltveränderungen (WBGU), dass die wirtschaftlichen Aktivitäten der Menschheit die planetaren Grenzen überschreiten und ihre kohlenstoffbasierte Wachstumsökonomie das Erdsystem mit geologischer Wucht verändern (Anthropozän). Dramatische Umweltveränderungen wie der Anstieg der mittleren globalen Temperatur und der Anstieg des Meeresspiegels in Folge des Abschmelzens von Meereis und Gletschern, die Versauerung der Ozeane, eine hohe Aussterberate von Tier- und Pflanzenarten sowie die Degradation und Versteppung von Landflächen gefährden die „Fähigkeit des Planeten, die Lebensgrundlage für künftige Generationen bereitzustellen" (WBGU 2011, 42). Weiterhin folgen wir der Forderung des WBGU nach einer „Großen Transformation": „Die Gesellschaften müssen auf eine neue ,Geschäftsgrundlage' gestellt werden. Es geht um einen neuen Weltgesellschaftsvertrag für eine klimaverträgliche und nachhaltige Weltwirtschaftsordnung" (WBGU 2011, 1-2, Kursiv. i. Orig.).

Doch obwohl bereits seit den 1970er Jahren von renommierten Institutionen auf die Grenzen des Wachstums hingewiesen wird (Meadows et al. 1972), auf dem „Erdgipfel“ von Rio 1992 Nachhaltigkeitsziele von praktisch der gesamten Menschheit unterschrieben wurden und 2015 die Vereinten Nationen 17 "Sustainable Development Goals“ verabschiedet haben, ist eine Trendumkehr weg von Rohstoff-Extraktivismus und emissionsreichem Verbrauch fossiler Brennstoffe hin zu nachhaltiger Produktion und Konsumption nicht zu verzeichnen. Selbst im Angesicht einer existenziellen Bedrohung - nämlich einer sich abzeichnenden „Klimatragödie“ , die ein „,kurzfristiges Aussterben“ nicht nur vieler Tier- und Pflanzenarten, sondern auch der eigenen Art zur Folge haben kann (Bendell 2018) - wirtschaftet die Menschheit im Großen und Ganzen weiter wie bisher, und zwar mit Wachstum als oberstem Ziel. Es stellt sich die Frage: Warum? 
Man kann dies wie Bendell (ebd.) mit psychologischen Prozessen der Leugnung von Realitäten zum Ziel der Aufrechterhaltung von Identitätskonstruktionen, internalisierten Werten, Lebensstilen und Lebenszielen erklären, im Grunde mit der Theorie der Vermeidung von kognitiver Dissonanz. Wer die Gefahr nicht (wirklich) wahrnimmt, muss auch nicht (wirklich) etwas dagegen tun - zumal es der „blinde Fleck“ jeder Kultur sei, sich ihre eigene Zerstörung und ihr eigenes Aussterben nicht vorstellen zu können (ebd., 15). Neben der Vermeidung kognitiver Dissonanz erwähnt Bendell einen zweiten Faktor, der die Bewusstwerdung des Problems erschwert und der mit Politischer Ökonomie verbunden ist:

"The politically permissible scientific consensus is that we need to stay beneath 2 degrees warming of global ambient temperatures, to avoid dangerous and uncontrollable levels of climate change, with impacts such as mass starvation, disease, flooding, storm destruction, forced migration and war. That figure was agreed by governments that were dealing with many domestic and international pressures from vested interests, particularly corporations. It is therefore not a figure that many scientists would advise, given that many ecosystems will be lost and many risks created if we approach 2 degrees global ambient warming" (ebd., 9).

Das Zwei-Grad-Ziel also, mit dessen Umsetzung sich viele Regierungen aus Angst vor Stimmenverlusten bei anstehenden Wahlen so offenkundig schwertun, ist mutmaßlich bereits das Ergebnis massiver Lobbytätigkeit von Wirtschaftsakteuren. Während also Verbraucher*innen von Wirtschaftsgütern offenbar Einschränkungen ihrer Konsumfreiheiten befürchten, wollen Produzent*innen von Waren und Dienstleistungen weiter ungehindert nach Gewinn streben: So könnte man umgangssprachlich zwei große Bremsklötze für eine "Große Transformation“ zur Nachhaltigkeit in Kurzform beschreiben.

Offensichtlich hängen diese Bremsklötze mit der Wirtschaftsform des Kapitalismus zusammen, dessen zentrales Merkmal der Zwang zur permanenten Gewinnsteigerung ist - nach Marx (1971, 170): G - W - G', aus Geld mach über die Produktion von Waren mehr Geld bzw. Kapital. Doch welches sind die ideologischen Grundlagen, die diese Wirtschaftsform stützen und reproduzieren? Und wie sollten sie von der Kommunikationswissenschaft adressiert werden, deren Forschungsgegenstand „öffentliche Kommunikation“ ja zentral ist "für die Aufrechterhaltung des Status quo - über die Verbreitung von Wissen, Normen, Werten und Ideologien“ (Krüger \& Meyen 2018, 351f.)? 


\section{Mentale Infrastrukturen des Kapitalismus}

„Ideologien“ werden hier begriffen als „Überzeugungssysteme, die praktische Konsequenzen haben. Sie wirken praktisch und sind ihrerseits Effekte einer bestimmten gesellschaftlichen Praxis." (Jaeggi 2009, 268) Sie legitimieren Macht- und Herrschaftsansprüche und/oder -strukturen, etwa indem sie diese unsichtbar machen oder als selbstverständlich erscheinen lassen. Haben Subjekte eine Ideologie akzeptiert, führt dies „zu einer teilweisen Aussetzung oder systematischen Verzerrung der Vernunftprüfung. Dadurch werden Herrschaft und Ausbeutung nicht als solche erkannt, sie vollziehen sich hinter dem Rücken der Subjekte und sind der Kritik entzogen.“ (Biskamp 2019, 69) Eine solche Naturalisierung von Herrschaft nennt Jaeggi (2009, 269) „Verselbstverständlichung“ bzw. "Selbstverständlichmachung".

Es erscheint notwendig, die Überzeugungssysteme, die hinter zerstörerischen gesellschaftlichen Praxen stehen bzw. mit diesen in einem wechselseitigen Bedingungs- und Ermöglichungszusammenhang stehen, möglichst genau zu identifizieren, um sie im Sinne einer "transformativen Kommunikationswissenschaft" wirksam durch Dekonstruktion und Kritik zu schwächen (Krüger \& Meyen 2018,351). Vielversprechend erscheint dieses Vorhaben, weil eine Ideologie dem davon durchdrungenen Individuum oft verborgen ist; ihre Benennung und Beschreibung sowie das Aufzeigen von inneren Widersprüchen oder von Diskrepanzen zwischen Sollen und Sein können wichtige Impulse zur Veränderung von Wahrnehmung und am Ende auch Verhalten sein.

Die Frage, welche Ideologie einer "Großen Transformation“ im Weg steht, wird in linken bis linksliberalen Milieus gern mit Verweis auf den seit den 1980er Jahren hegemonialen Neoliberalismus beantwortet (beispielhaft: Dietz 2018), also auf ein wirtschaftspolitisches Überzeugungssystem, das Unternehmen durch Deregulierung, Liberalisierung, Privatisierung, Steuersenkung und Sozialstaatsabbau zu stärken versucht. Jedoch darf nicht vergessen werden, dass Produktion und Konsumption im Globalen Norden schon seit dem Ende des Zweiten Weltkriegs exponentiell angestiegen sind (Steffen et al. 2015), mithin auch in Zeiten eines „,sozialdemokratischen Konsensus “ mit Keynesianischer (nachfrageorientierter) Wirtschaftspolitik. Tatsächlich sind sogar Teile der Linken mit einer Fixierung auf den nationalen Wohlfahrtsstaat seit Langem in das Steigerungs- und Ressourcenvernutzungsspiel des Kapitalismus und seine 
Externalisierungspraktiken (Lessenich 2016) verstrickt - Wachstum ist erwünscht, da es durch einen vergrößerten Kuchen Umverteilung ermöglicht. Mithin muss uns die Suche zu Überzeugungssystemen führen, die gleichsam „unterhalb“ des alles überwölbenden Kapitalismus als Wirtschaftssystem, aber "oberhalb“ der ordnungspolitischen Vorstellungen des Neoliberalismus angesiedelt, also klein(teilig)er als Kapitalismus, aber basaler als Neoliberalismus sind. Eine Kritik solcher Überzeugungssysteme würde Kapitalismuskritik nicht ersetzen, aber spezifizieren und treffsicherer und wirkungsvoller machen.

Hilfreich erscheint ein Blick in die Degrowth-Literatur, die verschiedene "Wachstumstreiber" auf vier verschiedenen Wirkungsebenen ausweist: auf der Ebene der gesellschaftlichen Leitbilder bestimmte mentale Infrastrukturen und Wachstumsgläubigkeit, auf der Ebene der Institutionen (verstanden nicht als Organisationen, sondern als Regelsysteme) etwa die Verzinsung von Krediten oder der Wettbewerb zwischen ökonomischen Akteuren, auf der Ebene der Unternehmen etwa die Unternehmensform der Aktiengesellschaften oder die Werbung, und auf der individuell-psychologischen Ebene das Streben nach materiellem Wohlstand oder Anerkennung durch Konsum (Petschow et al. 2018, 50). Der Psychologe und Nachhaltigkeitswissenschaftler Peter Schmuck identifiziert als Hindernisse für den ökologisch-sozialen Umbau unserer Gesellschaften zehn „Annahmen über unsere eigene psychische Natur sowie über die Gestaltung unseres Wirtschaftssystems und der Verteilungs- und Konsummuster", darunter: „Wir Menschen seien vor allem egoorientierte und wettbewerbsgetriebene Wesen“, "Andauerndes Wirtschaftswachstum sei notwendig" und „Konsum mache glücklich, viel Geld ermögliche viel Konsum und mache daher besonders glücklich" (Schmuck 2015, 15). Und der Sozialpsychologe Harald Welzer stellt die "mentalen Infrastrukturen“ in den Vordergrund, die in den letzten 200 Jahren durch eine "tiefe Industrialisierung" entstanden sind, insbesondere die Vorstellung, dass es „[i]n der expansiven Moderne (...) auch hinsichtlich der individuellen Existenz um Vergrößerung und Wachstum" (Welzer 2013, 58) geht, und die Idee, "dass alles immer verfügbar ist und zu sein hat" (ebd., 42f.).

Deutlich wird dabei, dass auf der Suche nach zu kritisierenden Ideologien nicht nur die Angebotsseite, also die ideologische Grundierung der Produktion von Waren und Dienstleistungen, sondern auch die Nachfrageseite, also die ideologische Grundierung des Konsums derselben, in den 
Blick zu nehmen ist. Es geht nicht nur um Überzeugungssysteme, mentale Infrastrukturen und Mindsets, die die Vorstandsvorsitzenden ferner Konzerne anleiten, sondern auch um jene, von denen möglicherweise wir alle - als Verbraucher*innen und als Wähler*innen - durchdrungen sind und die unsere „Weltbeziehung" konstituieren. Das betrifft letztlich "die Art und Weise, in der wir als Subjekte Welt erfahren und in der wir zur Welt Stellung nehmen" (Rosa 2016, 19) bzw. wie wir uns die Welt in einer "strukturell auf Steigerung und Reichweitenvergrößerung hin angelegten Sozialformation" (ebd., 722) aneignen.

Wir schlagen hier vor, dass die Transformationsresistenz unserer westlich-kapitalistischen Gesellschaften in zwei Paradigmen bzw. Mindsets begründet ist, die beide im 18. Jahrhundert wurzeln, also der Zeit, in der sich der Kapitalismus in der westlichen Welt durchzusetzen begann: die neoklassische Theorie der Wirtschaftswissenschaft, die das „mainstream economic paradigm“ (Göpel 2016) darstellt ${ }^{1}$ und auf der Produktionsseite für Nachhaltigkeitsfragen blind macht, und der "Romantische Konsumismus“, der Konsumptionssteigerungen und Konsumkultur in der breiten Bevölkerung begünstigt.

Die Neoklassik, der derzeit die weit überwiegende Mehrheit aller Ökonom*innen in der westlichen Welt anhängen (Heise und Thieme 2015, 159) und die in den wichtigsten Lehrbüchern für Mikro- und Makroökonomie als weitestgehend alternativlos dargestellt wird (van Treeck und Urban 2016; Rebhan 2017), kann als eine Eliten- bzw. Fach-Ideologie angesehen werden, die rational und differenziert auf sehr hohem Niveau ausformuliert ist. Reproduziert und verbreitet wird sie in Hörsälen und Lehrbüchern, in Fachjournal-Artikeln und auf wissenschaftlichen Konferenzen, in wirtschaftspolitischen Gutachten und in Parlamentsdebatten, in Wirtschaftsteilen von Prestigezeitungen und in Manager-Magazinen; ihre Träger*innen und Multiplikator*innen sind Volkswirtschaftsprofessor*innen und Betriebswirt*innen, Vorstände und Aufsichtsräte von Banken und Konzernen, Führungspersonal von zwischenstaatlichen Organisationen wie der Weltbank oder dem Internationalen Währungsfond,

1 Es mag an dieser Stelle für manche/n Leser*in befremdlich erscheinen, dass eine wissenschaftliche Theorie bzw. ein Paradigma als Ideologie klassifiziert bzw. denunziert wird. Im folgenden Abschnitt wird dies ausführlich begründet. Andere Kritiker*innen attestieren der Neoklassik sogar "Züge einer modernen Religiosität" (Butterwegge, Lösch und Ptak 2017, 27; Krätke 1999). 
Fiskalpolitiker*innen und Wirtschaftsjournalist*innen. Die öffentliche Arena, die die Kommunikationswissenschaft in Bezug auf die Neoklassik in den Blick nehmen müsste, ist demnach eine relativ kleine, aber wirkmächtige Arena des ökonomischen und wirtschaftspolitischen Elitendiskurses (akademische und/oder elitäre Encounter-Öffentlichkeiten, Fachmedien, Policy Papers usw.). Der Romantische Konsumismus dagegen ist eine Massen-Ideologie, die sich dadurch auszeichnet, dass sie gerade nicht auf hohem Niveau ausformuliert ist, sondern als unterschwellig-unbewusstes Überzeugungssystem vorwiegend im emotionalen, prärationalen Bereich wirkt. Reproduziert wird sie maßgeblich über die klassischen Massenmedien - und inzwischen auch über massenhaft genutzte Medien einer sich verschmelzenden Individual- und Massenkommunikation (soziale Netzwerkplattformen). Die Kommunikationswissenschaft wäre beim Aufspüren und Dekonstruieren derselben vor allem in klassischen medial hergestellten, allgemeinen Öffentlichkeiten (Werbung, fiktionale Unterhaltungsformate wie Filme und Serien, Journalismus) gefordert.

Im Folgenden werden beide Überzeugungssysteme skizziert und anschließend die diesbezüglichen Aufgaben einer ideologiekritischen Kommunikationswissenschaft konkretisiert.

\section{Die Neoklassische Ökonomik: Wesen und Kritik}

Die Neue Klassische Ökonomik (Neoklassik) 2 ist ein Produkt der vorherigen "Großen Transformation“, nämlich des fundamentalen gesellschaftlichen Wandels im 19. und 20. Jahrhundert beim Übergang von der feudalen Agrargesellschaft zur kapitalistischen Industrie- und Marktgesellschaft (Polanyi 1957). Die für diese Umwälzung zentralen geistigen Strömungen war zum einen die Aufklärung, durch die religiöse Überzeugungen zur Erklärung der Welt durch (natur-)wissenschaftliches Denken und Forschen abgelöst wurden, und zum anderen der

2 Die Neoklassik hat verschiedene Strömungen: eine makroökonomische, die oft als „New-Keynesian Economics“ oder „neoklassische Synthese“ bezeichnet wird, und eine mikroökonomische, die oft mit "Neoklassik“ gleichgesetzt wird. Die Ausführungen im Folgenden beziehen sich vor allem auf die Makroökonomik bzw. Volkswirtschaftslehre. 
Wirtschaftsliberalismus: Dieser setzte die Gewinnerzielung als Motiv des Wirtschaftens an die Stelle der Subsistenz und befreite sie von der kirchlichen Verdammung als Gier - und flankierte damit zugleich die politische Emanzipation des gebildeten und besitzenden Bürgertums von Adel und Klerus in wirtschafts- und ideenpolitischer Hinsicht.

Diese beiden Einflüsse, Aufklärung und Wirtschaftsliberalismus, sind wesentlich zum Verständnis der Neoklassik, weil diese sich fast ausschließlich um Märkte als Institutionen der Allokation von Ressourcen dreht und die Überlegenheit von Märkten gegenüber anderen Allokationsverfahren (z. B. staatlich gesteuerten) postuliert; und weil sie den Anspruch hat, Wirtschaft mit quasi-naturwissenschaftlicher Strenge objektiv zu beschreiben. So erklärte der französische Ökonom Léon Walras (18341910), einer der Gründungsväter des Paradigmas, seine „pure theory of economics is a science which resembles the physio-mathematical sciences in every respect" (Walras, zitiert nach Göpel 2016, 59).

Die Neoklassik versteht sich selbst als eine Weiterentwicklung der Klassischen Ökonomik von Adam Smith und anderen (Stichworte hier sind die "unsichtbare Hand des Marktes", "Laissez-faire“ und „Nachtwächterstaat") und rückt gegenüber der Klassik, die auf die Verteilung des „Wohlstands der Nationen“ fokussierte, den subjektiven Nutzen der Konsument*innen in den Fokus: Sie geht von einem rational handelnden, umfassend informierten und unablässig nach der Maximierung des eigenen Nutzens strebenden Individuums aus. Dieser "Homo oeconomicus" befriedigt seine Bedürfnisse mit dem Konsum von Produkten, wobei sich der Nutzen eines Produkts in seinem Preis und damit in der Zahlungsbereitschaft der Individuen ausdrückt. Auf perfekten, ungestörten Märkten pegeln sich dann Gleichgewichtszustände zwischen dem Angebot an Produkten und der Nachfrage nach selbigen ein, die „das größte Glück der größten Zahl“ garantieren - so das Leitprinzip der utilitaristischen Ethik von Jeremy Bentham (1748-1832), auf die Léon Walras seine ökonomische Gleichgewichtslehre gründete.

Definiert werden kann die Neoklassik als eine

„Theorie des Tausches (...), in der mit strengen mathematischen Methoden optimale Marktzustände in Gestalt von Gleichgewichtsmodellen konstruiert werden. Der perfekte Zustand einer Marktwirtschaft ist das Konstrukt einer vollständigen oder auch vollkommenen Konkurrenz, in der vielen Nachfragern viele Anbieter gegenüberstehen, die dabei über optimale Informationen und damit Markttransparenz verfügen, sodass ein gleichgewichtiges, effizientes Marktergebnis zustande kommt." (Butterwegge, Lösch und Ptak 2017, 27) 
Die Grundfrage der Neoklassik ist also die Erklärung der effizienten Allokation von knappen Ressourcen vor dem Hintergrund von präferenzund nutzentheoretischen Axiomen; ihre Methode ist die mathematische Funktionsalgebra, die darauf aufbauende Optimierungsrechnung sowie die Mengen- und Vektorrechnung (Beckenbach 2016, 83).

Zur Analyse von Angebot und Nachfrage auf einem Markt werden standardmäßig ",sowohl das gesamte Angebot als auch die gesamte Nachfrage nach einem gegebenen Gut als eine von einem vorgegebenen Preis für dieses Gut abhängige Funktion (grafisch als Angebots- bzw. Nachfragekurve) aufgefasst" (ebd., 86). Es wird sodann davon ausgegangen, dass bei Preisänderungen eine Anpassung der angebotenen und der nachgefragten Menge des Gutes entlang der Kurven stattfindet, bis sich beide Kurven schneiden: Dieser Schnittpunkt ist das Marktgleichgewicht (vgl. Abb. 1).

Laut Heise und Thieme $(2015,160)$ ist die Neoklassik gekennzeichnet von der „ausschließlichen Akzeptanz eines formalmathematisch deduktiven, positivistischen Reduktionismus (...), kombiniert (...) mit hochentwickelter Mikro- und Makroökonometrie oder auch experimentellen Versuchsanordnungen (...), wie sie aus den naturwissenschaftlichen Leitwissenschaften (Physik und Chemie) bekannt sind." Zentral in der neoklassisch geprägten Forschung und Lehre sind sogenannte DynamischStochastische Allgemeine Gleichgewichtsmodelle (DSGM). Das sind Modelle der Volkswirtschaft, in denen „die gesamtwirtschaftlichen Größen aus dem rationalen mikroökonomischen Kalkül einzelner Akteure und Akteurinnen abgeleitet werden“ (Dullien 2016, 201). Die Bedeutung der DSGM für die Disziplin ist so groß, dass - nach Ansicht einiger Beobachter*innen - sie es sind, denen im eigentlichen Sinn die vielstimmige Kritik an einem wirtschaftswissenschaftlichen "Mainstream" oder einer "Orthodoxie“ gilt: Heise und Thieme $(2015,157)$ sprechen vom „DSGMParadigma“ bzw. vom „DSGM-Mainstream“, der sich aus der Neoklassik und dem Neokeynesianismus zusammensetzt.

Zwar thematisieren die maßgeblichen Volkswirtschaftslehrbücher (etwa von Paul A. Samuelson oder von Gregory Mankiw) tatsächlich auch John Maynard Keynes, der für staatliche Interventionen in die Wirtschaft zum Zweck der Nachfrage-Stimulierung steht. Doch wird der Neokeynesianismus (der nur noch wenig mit der ursprünglichen Lehre von Keynes zu tun hat) in die Mainstream-Ökonomik so integriert, als ob es sich um 
einen „Sonderfall der neoklassischen Gleichgewichtslehre" handelt; hier liegt also „Domestizierung“ bzw. „Vereinnahmung“ von Keynes durch die Neoklassik vor, der eigentlich ein „Theoretiker der Instabilität und des Ungleichgewichts" von Märkten war (ebd., 179). Daher wird hier am Begriff „Neoklassik“ als Synonym für das „mainstream economic paradigm“ (Göpel 2016) bzw. die „ökonomische Lehrbuchwissenschaft" (Bäuerle 2017) festgehalten.

\section{Abb. 3-7}

Das Gleichgewicht von Angebot und Nachfrage

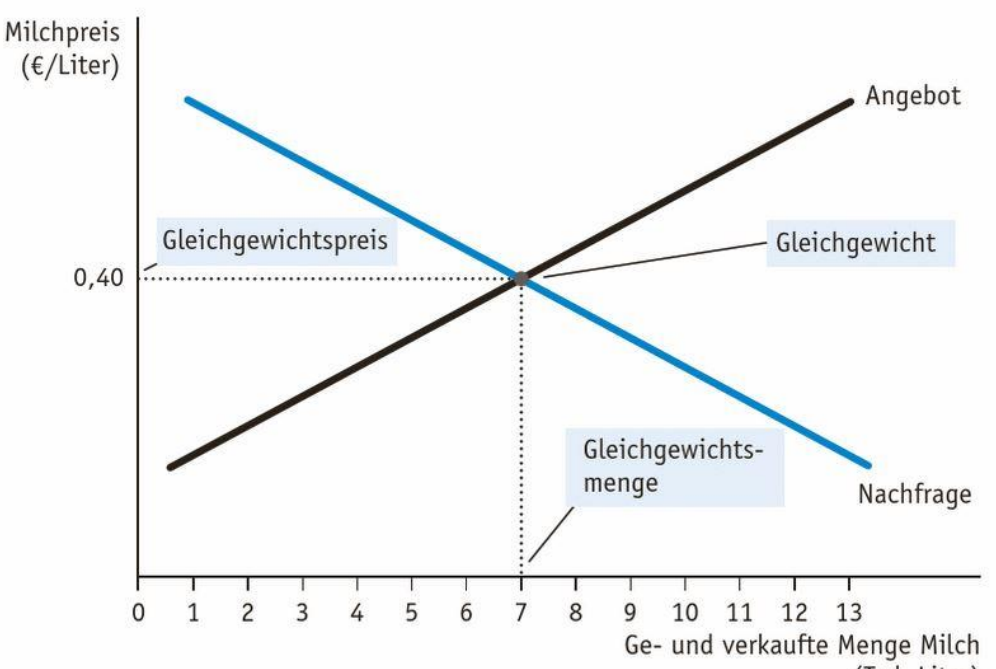

(Tsd. Liter)

Das Gleichgewicht findet man da, wo sich Angebots- und Nachfragekurve schneiden. Beim Gleichgewichtspreis entspricht die angebotene Menge der nachgefragten Menge. Hier beträgt der Gleichgewichtspreis 40 Cent pro Liter Milch: Zu diesem Preis sind die Verkäufer bereit, 7.000 Liter Milch anzubieten, und die Käufer fragen 7.000 Liter Milch nach.

Abb. 1: Beispiel für eine neoklassische Marktanalyse aus dem aktuell weltweit führenden Lehrbuch für Volkswirtschaft (Mankiw und Taylor 2018, 77). 
Was ist nun aus ideologiekritischer Perspektive - mit einer "Großen Transformation" zur Nachhaltigkeit als Fluchtpunkt - an der Neoklassik zu bemängeln? In die öffentliche Kritik geriet die Mainstream-Ökonomik spätestens nach der Weltfinanzkrise 2008 und der sich anschließenden Eurokrise ab 2010, da es ihr nicht gelungen war, diese Ereignisse (genauso wenig wie schon die Weltwirtschaftskrise 1929) vorherzusagen. Die mangelnde Prognosefähigkeit der DSGM ist eine Folge der Realitätsferne des neoklassischen Paradigmas, das „ontologisch auf einen Selbstregulierungsoptimismus [bezüglich des Marktes] festgelegt ist und sich fast komplett auf allokative Fragestellungen kapriziert, statt Stabilitätsbedingungen und -risiken zu hinterfragen" (Heise und Thieme 2015, 157f.).

Die ökonomische Lehrbuchwissenschaft hinkt dem Stand der Verhaltensforschung, der Neurowissenschaften oder der Forschung zur Dynamik komplexer Systeme hinterher. Allenfalls in der Forschung wird beschränkte Rationalität der Individuen oder unvollständige Information konzediert, ohne jedoch den Kern der Neoklassik in Frage zu stellen. Diese überträgt im Grunde Prinzipien der klassischen Mechanik auf die Wirtschaft: "Just as Newton believed that matter is made up of minute particles that bump off one another but are otherwise unchanged, so mainstream theory assumes that the economy is made up of unconnected individuals who interact by exchanging goods and services and money but are otherwise unchanged“ (Orrell 2010, zitiert nach Göpel 2016, 58). Die Vorstellung eines volkswirtschaftlichen Kreislaufs (vgl. Abb. 2) ist im Grunde eine Adaption des ersten Hauptsatzes der Thermodynamik (entdeckt im späten 18. Jahrhundert), laut dem Energie in geschlossenen Systemen nicht verlorengeht, sondern nur ihre Form wandelt; die Ökonomik hat Energie durch Kapital ersetzt, welches in Geld gemessen wird (Göpel 2016, 82). Schon der zweite Hauptsatz der Thermodynamik, nach dem in geschlossenen Systemen die Entropie ständig zunimmt und nur offene Systeme ihre Ordnung aufrechterhalten können (entdeckt im 19. Jahrhundert), hat es nicht mehr in die neoklassische Lehre geschafft. 


\section{Abb. 20-1}

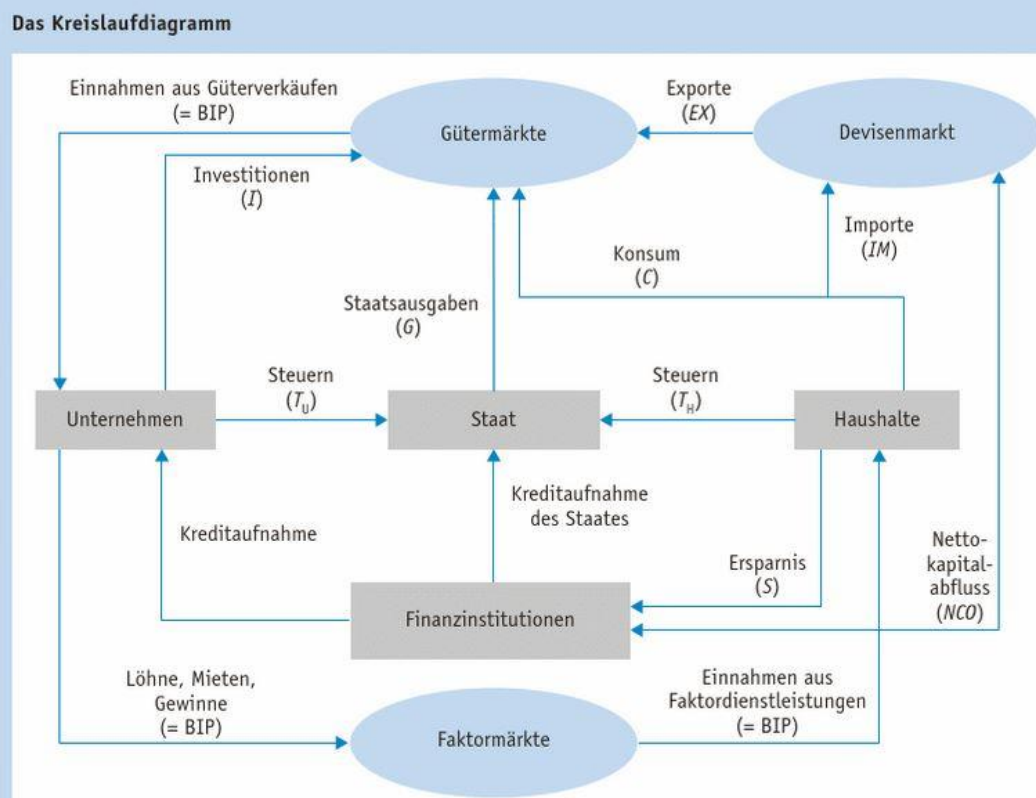

Haushalte kaufen Güter von den Unternehmen und die Unternehmen verwenden ihre Einnahmen aus den Verkäufen, um Löhne an die Beschäftigten, Miete oder Pacht an die Grundbesitzer und Gewinne an die Unternehmenseigner zu zahlen. Das BIP entspricht der gesamten Summe, die die Haushalte auf dem Gütermarkt ausgeben. Es entspricht ebenfalls den gesamten Zahlungen der Unternehmen an Löhnen, Mieten oder Pacht und Gewinnen auf den Faktormärkten. Abflüsse aus dem Kreislauf beinhalten Steuern, Ersparnisse und Ausgaben für Importe, während Staatsausgaben, Investitionen und Exportumsätze Zuflüsse zum Kreislauf darstellen.

Abb. 2: Kapital geht nicht verloren: Volkswirtschaftlicher Kreislauf im Lehrbuch nach dem Muster des Ersten Hauptsatzes der Thermodynamik (Quelle: Mankiw und Taylor 2018, 623).

Damit zusammenhängend weist die Neoklassik massive blinde Flecken im ökologischen und sozialen Bereich auf: Während der subjektive Nutzen eines Produkts, dargestellt durch den Preis, eine zentrale Kategorie ist und Menschen als unersättliche Maximierer ihres Eigennutzes erscheinen, wird nach den vielschichtigen (und keineswegs nur ökonomischen) Bedürfnissen von Menschen nicht näher gefragt. Dass Menschen etwa Befriedigung durch eine sinnvolle berufliche Tätigkeit erfahren können (also als Teilnehmer*innen am Produktionsprozess von Gütern), sieht die Neoklassik nicht: Nutzen entsteht vor allem durch Konsum (bzw. durch 
Einkaufen), und eine Konsumsteigerung ist gleichbedeutend mit einer Steigerung von Nutzen bzw. Bedürfnisbefriedigung (Göpel 2016, 60). Ebenso fehlt eine umfassende Betrachtung der natürlichen Mitwelt: So ist unterbelichtet, dass Naturressourcen für Menschen einen Wert außerhalb von Güterproduktion haben (z. B. als Erholungsgebiet oder als Bereitsteller von Lebensgrundlagen wie Trinkwasser oder sauberer Luft) und dass bei der Produktion eines Gutes neben dem Nutzen für den Konsumenten Schäden durch Verschmutzung oder Müll entstehen (Göpel 2016, 61f.). Umweltökonom*innen innerhalb des neoklassischen Paradigmas arbeiten zwar daran, die Umwelt über den Weg der Bepreisung in die neoklassischen Modelle $\mathrm{zu}$ integrieren. ${ }^{3}$ Jedoch kranken diese Versuche an der mechanistisch-additiven Grundstruktur der Basistheorie, die mit der Komplexität natürlicher Zusammenhänge im Gewebe des Lebens unvereinbar ist:

„However, the creation of markets for ecosystem services only works on single atomized units of capital, like a ton of $\mathrm{CO}$, and thus tells us little about the whole web of natural life. Living species interact and form complex ecosystems with balancing feedback loops and food chains. Thus, overexploiting one type of resource, or condemning one species to extinction may seem harmless. But without an understanding of these intricate relationships, we may create a ,missing link,' glut or shortage in the dynamic reproduction circuits of basic life support systems like water supply, pollination patterns or soil fertility" (Göpel 2016, 85).

Tatsächlich, und dies scheint neben einem veralteten Weltbild das Hauptproblem zu sein, ist die Mainstream-Ökonomik eine Sozialwissenschaft, die in der Forschung zwar empirisch arbeitet, aber ihre Daten typischerweise in einem engen Theorierahmen interpretiert, anstatt zu versuchen, reale Phänomene der Wirtschaft mit Hilfe verschiedener Theorieansätze zu verstehen. In der universitären Lehre besteht sie vor allem in der $A n-$ wendung vorgegebener, grundlegend im späten 19. und frühen 20. Jahrhundert entwickelter Denkwerkzeuge auf verschiedene ökonomische (und nicht-ökonomische) Sachverhalte. Hier versteht sich die Ökonomik "als eine erfahrungsunabhängige Wissenschaft", die sich „,nicht durch einen einheitlichen Gegenstand (,Wirtschaft'), sondern durch eine einheitliche Denkmethode" (Bäuerle 2017, 263, Hervorh. i. Orig.) definiert. Ziel der wissenschaftlichen Tätigkeit ist das Erstellen eines abstrakten, stringenten

3 Der Emissionshandel ist ein Beispiel für einen marktbasierten Ansatz zur Lösung des Klimaproblems. 
Modells: „Ist es in diesem Sinne ausgereift, wird es nicht getestet, sondern angewandt" (ebd., 263f., Hervorh. i. Orig.). Es kann nicht an der Realität scheitern, sondern nur an inneren Inkonsistenzen.

In Lehrbüchern zeigt sich der Charakter der Neoklassik als idealistische, apriorische, nicht-empirische Wissenschaft, wenn von der „ökonomischen Perspektive“ oder der "volkswirtschaftlichen Denkweise“ die Rede ist, die auf „dauerhaften Wahrheiten“ gründe und deren „Denkwerkzeuge“ (Angebotskurve, Nachfragekurve, Gleichgewichtspreis, Präferenzen, Grenznutzen usw.) die Studierenden durch Wiederholungen und immer neue Anwendungsbeispiele einüben sollen (Graupe 2016, 22). Hier zeigt sich, dass wir es mehr mit einer festgefügten Weltanschauung als mit einem Erkennen-Wollen zu tun haben; und diese Weltanschauung bzw. dieses „Einfinden in eine ungestörte, entkontextualisierte und enthistorisierte Perspektive“ muss im Studium „durch unablässiges Einüben und Anwenden ein- und desselben Denkschemas“ (Bäuerle 2017, 267) trainiert werden.

Hier wird nicht die Auffassung vertreten, dass jede Theorie im Sinne von Karl Poppers Kritischem Rationalismus falsifizierbar sein muss; allein die Tatsache, dass DSGM schwer oder gar nicht falsifizierbar sind (Dullien 2016, 204f.), macht die Neoklassik noch nicht zur Ideologie. Auch Gesellschafts- bzw. Universaltheorien wie Luhmanns Systemtheorie, Bourdieus Feldtheorie oder der Marxsche Historische Materialismus sind - im Unterschied zu Theorien mittlerer Reichweite - nicht falsifizierbar bzw. kaum empirisch überprüfbar (Scholl 2016, 94ff.), stellen selbst ebenso wie die Neoklassik „Denkwerkzeuge“ und „Perspektiven“ bereit. Die Neoklassik entscheidet jedoch eine politische Frage („Ist der Markt das beste Allokationsverfahren?") vor, indem sie erst nach ihrer positiven Beantwortung mit der Wissenschaft beginnt. Die Antwort auf diese wirtschaftspolitische Frage, die im Angesicht der laufenden Klimatragödie und der grassierenden Zunahme von sozialer Ungleichheit zentral ist, stellt ein unausgesprochenes, verdecktes Axiom dar. Dieses steckt - um eine Baum-Metapher zu verwenden - sozusagen unter der Erde in der Wurzel des Paradigmas, von dem nur der Stamm (die in den Lehrbüchern als "dauerhafte Wahrheiten“ ausgesprochenen Axiome) und die Baumkrone (die Verästelungen der Theorie etwa in aktueller Forschung) sichtbar sind (vgl. Bäuerle 2017, 258). Die Dominanz von Märkten wird so naturalisiert bzw. unsichtbar gemacht, sie erscheint als nicht hinterfragbar. Luhmanns Systemtheorie tut nichts Vergleichbares, sofern man die Arbeitsteilung 
bzw. die funktionale Differenzierung der Gesellschaft nicht als politische Frage auffasst; Bourdieus Feldtheorie tut dies auch nicht. Der Marxismus als heterodoxe Ökonomik tut dies in der Analyse und Kritik des Kapitalismus zunächst nicht; er tut es als Ideologie dann, wenn er Geschichte teleologisch als Bewegung hin zur Auflösung von Klassengegensätzen in einer Diktatur des Proletariats erzählt.

Daher kann man mit einigem Recht behaupten, die Neoklassik als ökonomische Lehrbuchwissenschaft betreibe "mathematisch rationalisierte Ideologieproduktion" (Beckenbach 2016, 96). In ihrer orthodoxen Form ist sie eine Legitimationstheorie für die Herrschaft des Marktes und seiner Profiteure, ähnlich wie es ihr Antipode, der orthodoxe Marxismus, für die Herrschaft kommunistischer Parteien war (bzw. im Fall von Nordkorea noch ist). Sie ist - um noch einmal mit Jaeggi $(2009,268)$ zu sprechen - ein Überzeugungssystem, das praktische Konsequenzen hat und Herrschaftsansprüche verselbstverständlicht.

Die verdeckten ideologischen Elemente des Paradigmas mögen historisch mit den Überzeugungen der damaligen wirtschaftsliberalen Intellektuellen zusammenhängen, die während der letzten „Großen Transformation" die Durchsetzung einer "freien“ Marktgesellschaft gegen ein überkommenes feudales System unterstützen wollten. Dass sich seit dem Zweiten Weltkrieg die gesamte Disziplin auf das neoklassische Paradigma verengt hat, hängt aber wohl mit wissenschaftsendogenen wie exogenen Faktoren zusammen. Heise und Thieme $(2015,182)$ erklären die erfolgte "(Ab-)Schließung des Wissenschaftssystems" unter neoklassischen Vorzeichen mit zwei Faktoren. Zum einen mit der „Festlegung paradigmatischer Standards" im Bestreben, als reife Disziplin anerkannt zu werden - Theorie-Monismus werde als „Ausweis der Reife und der ,Normalität' im Wissenschaftsgeschäft" verstanden. Zum anderen habe die reale Macht des Marktes vor allem in den USA (von wo aus die wirtschaftswissenschaftlichen Standards in alle Welt exportiert wurden) dazu geführt, dass sich eine entsprechende Strömung durchgesetzt hat: „(..) die Art und Weise, wie eine Gesellschaft ihre Basisinstitutionen (z. B. den Markt) kulturell umhegt, [wirkt] auf die Akzeptanz theoretischer Ansätze und paradigmatischer Zugänge - völlig unabhängig von deren faktischer Erklärungskraft - zurück“ (ebd., 178). Elsner (2016, 44f.) wird noch deutlicher, wenn es um die Frage geht, "wie es möglich sein kann, dass ein Paradigma seine Erschöpftheit, offensichtliche funktionale 
Unbrauchbarkeit und sein Versagen gegenüber der Realität nicht nur überlebt, sondern dominantes Paradigma bleibt und sich sogar offensiver als zuvor personell in der Academia ausbreitet": Er sieht wissenschaftsexogene Einflüsse (vor allem durch Drittmittelaufträge von der Privatwirtschaft und vom Staat, Politikberatung und Wechselbeziehungen mit den etablierten Medien) und resümiert, der neoklassische Mainstream habe

„in letzter Instanz die außerwissenschaftliche Funktion zu erfüllen (...), die führende Ideologie und Rhetorik für die aktuellen ökonomischen, sozialen und politischen Machtstrukturen und den System-Status-quo bereitzustellen. Kein anderes als das neoklassisch-neoliberale ökonomische Paradigma und keine andere wissenschaftliche Disziplin als diese Ökonomik ist in der Lage, diese Funktion zu erfüllen; daher die ihr durch , die Eliten' zugewiesene quasi-monopolistische, rhetorisch-definitorische Machtposition in Massenlehre und autoritativen Lehrbüchern." (ebd., 45f.)

Für eine wünschenswerte "Große Transformation“ zur Nachhaltigkeit ist die Dominanz eines solchen marktfixierten wirtschaftswissenschaftlichen Paradigmas fatal, wenn sie verhindert, dass eine längerfristige politische (demokratisch kontrollierte) Planung und Steuerung nötiger Teilprozesse überhaupt in den Bereich des Denkbaren rückt.

\section{Der Romantische Konsumismus: Wesen und Kritik}

Der israelische Universalhistoriker Yuval Noah Harari erwähnt in seinem Weltbestseller Eine kurze Geschichte der Menschheit eine die heutige Gesellschaft prägende Ideologie: den „Romantischen Konsumismus“, eine Mischung aus „zwei zentralen Ideologien der Moderne: Romantik und Konsumismus" (Harari 2015, 147). Als Beispiel erwähnt er „den verbreiteten Wunsch, im Ausland Urlaub zu machen. Dieser Wunsch ist weder natürlich noch naheliegend. Das Alphamännchen einer Schimpansenhorde käme nie auf den Gedanken, sich erst zu verausgaben, um dann im Territorium einer anderen Schimpansenhorde auszuspannen." (ebd.) Auch ein reicher Mann im alten Ägypten „hätte nie auch nur im Entferntesten daran gedacht, eine Beziehungskrise mit einer romantischen Reise nach Babylon beizulegen. Vermutlich hätte er seiner Frau das prächtige Grabdenkmal gebaut, von dem sie immer geträumt hat" (ebd., 148).

Mit diesen amüsanten Kontrasten arbeitet Harari heraus, dass unsere Sehnsüchte nach exotischen Urlaubserlebnissen, neuesten technischen 
Gerätschaften oder einem spirituell erleuchtenden Wochenendworkshop in Kundalini-Yoga weder überzeitliche Wünsche noch ureigenster Ausdruck unserer individuellen Persönlichkeit sind. Sie zeigen unsere gelungene Integration in ein Überzeugungssystem der westlichen Kultur, das uns Glück durch eine Vielzahl und Vielfältigkeit von Erlebnissen (Romantik) sowie Glück durch die Inanspruchnahme von Waren und Dienstleistungen (Konsum) verheißt.

Auch wenn Harari für diese Ausführungen keine Referenz angibt, bezieht er sich offensichtlich auf den Kultursoziologen Colin Campbell, der in seiner 1987 erschienenen Studie The Romantic Ethic and the Spirit of Modern Consumerism (die Ähnlichkeit zu Max Webers The Protestant Ethic and the Spirit of Capitalism ist konstitutiv) der Entstehung einer Konsumethik nachspürt, die aus der kulturgeschichtlichen Epoche der Romantik (ab dem späten 18. Jahrhundert) herrührt und Grundlage der modernen Konsumgesellschaft bildet. Beide Geisteshaltungen, Webers Produktionsund Campbells Konsumethik, haben ihre Ursprünge in der Industriellen Revolution und im Protestantismus. Beide waren notwendig für den wirtschaftlichen Umbruch im 18. und 19. Jahrhundert, beide wirken in einer Art Wahlverwandtschaft („elective affinity“) zusammen und bis heute nach (Campbell 2018, 305).

Laut Campbell förderte die romantische Ethik die Entwicklung des Konsums, indem sie die moralischen Barrieren aufhob, die den Konsum verhinderten (Puritanismus, Rationalismus, Utilitarismus). Individuum und Individualismus wurden aufgewertet, dem Ausdrücken von Gefühlen und Affekten, Kreativität und Rebellion neue Bedeutung und Anerkennung beigemessen, sodass die romantische Ethik dazu beitrug, den Konsum zu legitimieren.

Campbell entwickelt den Typus des „modern autonomous imaginative hedonist" bzw. "self-illusory hedonist", dessen Entstehung er in der englischen Mittelschicht des 18. Jahrhunderts beobachtet. Ein solcher moderner Hedonist strebt nicht nur nach Genuss, sondern vor allem nach Selbstverwirklichung, was von seinen Träumen und seiner Fantasie bestimmt wird. Er weist seinem Handeln und so auch seinem Konsum Bedeutungen zu. Dies geschieht selbstbestimmt, wird aber teilweise durch das soziale Umfeld und Medien beeinflusst - immer jedoch entsprechend seiner in der Kultur verankerten Wert- und Glaubensvorstellungen. Der „romantische Konsument" sehnt sich nach immer neuen, noch nicht 
vertrauten Produkten oder Dienstleistungen, da diese die Möglichkeit versprechen, sich an noch nicht gemachten (sinnlichen) Erfahrungen zu erfreuen. Da die Realität nicht an die Imaginationen heranreicht, wird die Sehnsucht niemals befriedigt und es werden immer neue (diffuse) Sehnsüchte generiert (Campbell 2018, 140-143).

Campbell bettet seine Postulate in soziologische Theorien ein; so führt die "motivated action theory“ über monokausale Erklärungen von Konsument*innenverhalten hinaus. Handlungsentscheidend sind nicht nur "harte“ ökonomische Faktoren wie Preisentwicklungen, sondern auch Werte- und Glaubenssysteme. Nach Campbell ist der Konsument nicht rational oder utilitaristisch; Gefühle und Sehnsüchte spielen ebenso eine Rolle wie die Konstruktion von Mehrwerten (Zuschreibung von Bedeutungen) und Identitäten. Indem Campbell darlegt, dass die Sehnsucht der Konsument*innen prinzipiell nicht durch Konsum befriedigt wird, wirft er außerdem ein Licht auf den Motor, der das kapitalistische Wachstumssystem am Laufen hält.

Seine kulturgeschichtliche Analyse beschränkt sich auf das 18. Jahrhundert, doch inspiriert zu seinen Nachforschungen wurde Campbell durch eigene Beobachtungen während der 1960er Jahre, als ein exponentieller Anstieg von Produktion und Konsum in der westlichen Welt mit einem Ausbruch von „romantic fever“ (Campbell 2018, 29) zusammenfiel. Bereits vorher war von interessierter Seite Konsum systematisch befördert worden: durch immer professionelleres Marketing, durch psychologisch fundierte Werbung, die es verstand, immer neue Wünsche zu wecken, und durch die Einführung von Konsumentenkrediten (in den USA ab den 1920er, in Westeuropa ab den 1950er Jahren), die „eine drastische Verkürzung der Zeitspanne zwischen Bedürfnis und Befriedigung“ ermöglichten (Welzer 2013, 47). Die Strategie der gezielten romantischen Aufladung von Waren und Dienstleistungen illustriert ein Zitat des amerikanischen Marketingexperte Victor Lebov aus den 1950er Jahren: „Unsere ungeheuer produktive Wirtschaft verlangt, dass wir den Konsum zu unserem Lebensstil und den Kauf und die Nutzung von Gütern zu einem Ritual machen, dass wir unsere spirituelle Befriedigung und die Erfüllung unseres Selbst im Konsum suchen." (zitiert nach Welzer 2013, 47f.).

Um dies klarzustellen: Konsum im Sinne von „Verbrauch" oder "Gebrauch" von bestimmten Objekten (Lebensmittel, Kleidung etc.) ist eine lebensnotwendige Tätigkeit, die niemand vermeiden kann. Konsumismus als Kultur bzw. Ideologie hingegen meint, dass dem Konsum eine 
(etwa gegenüber der Arbeit) gesteigerte Bedeutung für das soziale $\mathrm{Zu}$ sammenleben und die eigene Identität zugeschrieben wird. Für Hochstrasser $(2013,58)$ liegt eine konsumistische Kultur dann vor, wenn die Subjekte „ihre Selbstanerkennung und die soziale Anerkennung, welche ihnen die anderen zollen, zunehmend aus dem Konsum [ziehen]. Die Menschen werden einander anerkennenswert durch ihre Aktivitäten in der Konsumsphäre: Wichtig wird, was jemand wie, wie oft und mit wem konsumiert."

Die implizite Botschaft des Konsumismus lautet in Kurzform: „Sinn (...) kann man kaufen.“ (Welzer 2013, 46) Die Ideologiekritik daran kann man ebenso kurz halten: Sinn kann man nicht kaufen. In Haben oder Sein hat Erich Fromm (1979) hat diese Konsumkritik aus psychoanalytischer Sicht ausgearbeitet. Auch die Sozialpsychologie zeigt, dass das Streben nach Anerkennung durch relative Besserstellung (positionaler Konsum) bzw. durch soziale Symbolik materieller Güter (Statuskonsum) nicht zu erfülltem Lebenssinn und Zufriedenheit führt, sondern geradewegs in eine "hedonische Tretmühle“, in dem sich die Subjekte in einer "Konsumierendenkonkurrenz" aufreiben, um im Vergleich zu ihren Mitmenschen nicht ins Hintertreffen zu geraten (Hochstrasser 2013, 71; Göpel 2016, 67-76).

Dessen ungeachtet steigt der Konsum auch und vor allem in der von galoppierender Individualisierung und Digitalisierung geprägten Postbzw. Spätmoderne: Campbell $(2018,7)$ spricht im Vorwort einer Neuausgabe seiner Studie vom heutigen "hyper-consumerism", der von ausgefeilten Techniken von Werbung („high-pressure advertising“) befeuert wird und von Marketing, das mit großen Datensätzen der Konsumentenforschung unterfüttert ist. Ein Ende des Romantischen Konsumismus ist nicht in Sicht.

\section{Aufgaben einer transformativen Kommunikationswissenschaft}

Programm einer transformativen, d. h. im Sinne einer "Großen Transformation" engagierten Kommunikationswissenschaft ist es, erstens zu fragen, welche mit öffentlicher Kommunikation verbundenen Akteure, Strukturen und Prozesse Transformation zur Nachhaltigkeit behindern, 
um sie durch Dekonstruktion und Kritik zu schwächen; und zweitens zu fragen, welche mit öffentlicher Kommunikation verbundenen Akteure, Strukturen und Prozesse Transformation begünstigen, um sie durch fachöffentliche und öffentliche Aufmerksamkeit sowie durch Wissensproduktion zu stärken (Krüger \& Meyen 2018, 351f., Krüger 2019).

Als Transformationsbremser können Vertreter*innen der Neoklassischen Ökonomik gelten sowie Strukturen und Prozesse, die die Aufrechterhaltung dieses Paradigmas trotz massiver Kritik aus der Gesellschaft sowie aus der Wirtschaftswissenschaft selbst ${ }^{4}$ ermöglichen. Mit der Feldtheorie von Bourdieu als Fundierung haben Heise und Thieme (2015) die Kämpfe im „Machtfeld“ der Wirtschaftswissenschaft in Deutschland und das Ringen der Vertreter*innen von Orthodoxie und Heterodoxie um ökonomisches, soziales, kulturelles und symbolisches Kapital bereits beschrieben. Der Beitrag der Kommunikationswissenschaft könnte es sein, eine Theorie der Vermachtung von Fachöffentlichkeiten zu entwickeln die übrigens auch auf die eigene Disziplin angewandt werden könnte (vgl. Scheu 2012, Meyen 2017 und Krüger 2019 [darin das Vorwort zur 2. Auflage]). Eine solche Theorie könnte sich auf die Theorie des kommunikativen Handelns von Habermas beziehen: Hier ist die Rede von einer nicht-vermachteten Öffentlichkeit, die aus nicht-institutionalisierten zivilgesellschaftlichen Zusammenhängen (lebensweltliche Peripherie) heraus Probleme identifiziert und thematisiert, und einer vermachteten Öffentlichkeit nahe des System-Zentrums, "die von staatlichen und sozialen Institutionen zu Legitimationszwecken strategisch hergestellt wird“ (Brosda 2008, 313). Habermas böte mit seiner Unterscheidung zwischen dem Ideal eines quasi-natürlichen, verständigungsorientierten kommunikativen Handelns und einem strategischen Handeln, das von den Steuerungsmedien Geld und Macht beeinflusst ist und als deformiert und

$4 \quad$ Forderungen nach Paradigmenvielfalt, methodischem Pluralismus und mehr Interdisziplinarität in der Wirtschaftswissenschaft wurden zum Beispiel von der internationalen Studierendeninitiative ISIPE (International Students Initiative for Pluralism in Economics) und dem deutschsprachigen Netzwerk Plurale Ökonomik erhoben (van Treeck und Urban 2016, 11f.). Eine Liste weiterer Initiativen, Appelle und Manifeste findet sich bei Heise und Thieme $(2015,158)$. Ironischerweise war an einem "Aufruf für eine pluralistische und gründliche Ökonomik" aus dem Jahr 1992 auch Paul A. Samuelson beteiligt, „einflussreichster Lehrbuchautor aller Zeiten“ (vam Treeck 2016, 136), der in den 19 Auflagen seines in 41 Sprachen übersetzten Standardwerks Economics seit 1948 die Homogenisierung der Ökonomik maßgeblich vorangetrieben hatte. 
defizitär gesehen wird (vgl. Baugut 2017, 133-137), auch einen ideologiekritischen Zugang zu Kommunikaten und Kommunikationsprozessen in der (Wirtschafts-)Wissenschaft.

Eine Theorie zur Vermachtung von Fachöffentlichkeiten könnte sich auch anlehnen an das Propagandamodell von Herman und Chomsky (2002), das in den großen Medien eine Verengung des Themen- und Meinungsspektrums im Interesse der herrschenden politischen und wirtschaftlichen Eliten postuliert und mit einer Reihe von „Filtern“ erklärt (siehe die Beiträge von Kim Kristin Mauch und Holger Pötzsch in diesem Band). Eventuell wirken am Machtpol der Wirtschaftswissenschaften ganz ähnliche Filter (etwa "Antikommunismus als Kontrollmechanismus“), vielleicht aber auch andere Filter oder pressure groups als am Machtpol der journalistischen Massenmedien. Damit zusammenhängend könnte auch der Umgang des wissenschaftlichen Machtpols mit Heterodoxie und mit innerfachlichen Protestbewegungen (und damit die Frage der Sozialen Kontrolle) konzeptualisiert werden, etwa mit Hilfe der medienbezogenen Ansätze „Protest-Paradigma“ und "Guard Dog Perspective“ (vgl. Krüger 2019, 68-71).

Mit einer derartigen kommunikationstheoretischen und herrschaftskritischen Fundierung könnten Lehrbücher und innerwissenschaftliche Debattenbeiträge, Forschungsartikel in peer-reviewed journals, Selbstverständnispapiere von Ökonom*innen-Vereinigungen wie dem Verein für Socialpolitik, Jahresgutachten des Sachverständigenrats zur Begutachtung der gesamtwirtschaftlichen Entwicklung, Empfehlungen wissenschaftlicher Beiräte von Bundesministerien, Kommentare und Gastbeiträge in Wirtschaftszeitungen, Protokolle der zweijährlichen Treffen der Mont Pelèrin Society oder wirtschaftspolitische Parlamentsdebatten analysiert werden. Von besonderem Interesse wäre neben der Identifikation und Dekonstruktion ideologischer Aussagen das Aufzeigen von Akteursnetzwerken über die Wirtschaftswissenschaft hinaus, also der Verflechtungen von Fachvertreter*innen mit Eliten aus Politik und Wirtschaft (im Sinne einer power structure research nach Mills 1956), sowie der kommunikative Umgang des wirtschaftswissenschaftlichen Machtpols mit Dissentern im Fach und Trägern von Protest und Pluralismus-Forderungen.

Die Naturalisierung des Wachstumszwangs findet sich auch im Wirtschaftsjournalismus; so ergab eine Analyse der Meinungsbeiträge in der Zeit, im Spiegel und in der FAZ von 1949 bis 2009, dass die tonangebenden 
Wirtschaftsjournalist*innen mehr oder weniger die Standard-Ökonomie und ihre Prämissen reproduzierten (Knauß 2016). Eine genauere Analyse der Bildungswege von Wirtschaftsjournalist*innen, von Einflüssen aus dem Studium der Betriebs- und Volkswirtschaftslehre oder von anderweitigen Verbindungen und Wechselwirkungen mit der Ökonomik wäre von Interesse. Kritische Analysen der Politischen Ökonomie von Massenmedien (wie jüngst Ferschli, Grabner und Theine 2019) oder Social-Media-Plattformen (etwa Sevignani 2016) sind ohnehin grundlegende Aufgaben der Kommunikations- und Medienwissenschaft.

Behindert wird Transformation auch von Akteuren, Strukturen und Prozessen, die dazu beitragen, den Konsum von Waren und Dienstleistungen zu romantisieren: Die Beschreibung entsprechender Strategien und die Dekonstruktion solcher Werbebotschaften sollte eine ideologiekritische Werbeforschung leisten (Beispiele: Williamson 2000; Leiss, Kline und Jhally 1986). Der Transfer der Ergebnisse in die Bevölkerung und die Vermittlung entsprechender Kompetenzen an Schüler*innen und Erwachsene ist hier besonders wichtig, wenn solche Forschung selbst nicht schon kollaborativ und partizipativ-emanzipatorisch (als citizen science) angelegt ist.

Schließlich gilt es in Sachen Konsumismus, "systemüberwindende Perspektiven" (Hochstrasser 2013, 330-349) mit kommunikationswissenschaftlichen Mitteln zu flankieren. Solche Ideen, die Transformation zur Nachhaltigkeit begünstigen, sind etwa eine Neudefinition des Begriffs "Arbeit", eine Reduktion der Warenmenge, die Etablierung von Suffizienz und Subsistenz als Ziel von Wirtschaftsaktivität, Produktionsverbote von Nonsens-Waren, Dekommodifizierung, Werbeverbote und eine Relokalisierung der globalisierten Ökonomie. Wie für entsprechende Ideen und alternative Praxen Öffentlichkeit hergestellt wird bzw. werden sollte und welche Rolle dabei Journalismus (Stichworte: Konstruktiver Journalismus, Verbraucherjournalismus), Öffentlichkeitsarbeit bzw. Public Relations oder soziale Netzwerkplattformen spielen: All dies können Themen einer transformativen Kommunikations- und Medienforschung sein. Dabei sollte diese übrigens ideologiekritisch und selbstreflexiv genug sein, um nicht wiederum neuen Ideologien zu erliegen (etwa einer Romantisierung von Selbstversorgung und Autarkie, zu der Teile der Degrowth-Bewegung neigen) und dadurch wiederum blind für wichtige gesellschaftliche Realitäten und Zusammenhänge zu werden. Denn nur 
mit unverstelltem Blick kann sie eine konstruktive Rolle in einer „Großen Transformation“ zur Nachhaltigkeit spielen.

\section{Literatur}

Bäuerle, Lukas. 2017. „Die ökonomische Lehrbuchwissenschaft - Zum disziplinären Selbstverständnis der Volkswirtschaftslehre“. Momentum Quarterly 6 (4): 252-270, https:// www.momentum-quarterly.org/ojs2/index.php/momentum/article/view/2478. Zugegriffen: 15. November 2019.

Baugut, Philip. 2017. Politiker und Journalisten in Interaktion. Einfluss des Medienwettbewerbs auf lokale politische Kommunikationskulturen. Wiesbaden: Springer VS.

Beckenbach, Frank. 2016. „Mathematisch rationalisierte Ideologieproduktion statt Marktanalyse. Zur Kritik der Denkform der neoklassischen (Lehrbuch-)Mikroökonomie“. In Wirtschaft neu denken. Blinde Flecken der Lehrbuchökonomie, herausgegeben von Till van Treeck und Janina Urban, 82-97. Berlin: iRights Media, http://www.fgwnrw.de/fileadmin/user_upload/Blinde_Flecken_der_Lehrbuchoekonomie_klein.pdf. Zugegriffen: 15. November 2019.

Bendell, Jem. 2018. „Deep Adaptation: A Map for Navigating Climate Tragedy”. IFLAS Occasional Paper 2, https://jembendell.com/2019/05/15/deep-adaptation-versions/. Zugegriffen: 15. November 2019.

Biskamp, Floris. 2019. „Ideologiekritik als Kritik systematisch verzerrter Kommunikationsbedingungen. Zum ideologiekritischen Potenzial der Habermas'schen Theorie“. In Ideologie, Kritik, Öffentlichkeit. Verhandlungen des Netzwerks Kritische Kommunikationswissenschaft, herausgegeben von Uwe Krüger und Sebastian Sevignani, 66-84. Universität Leipzig: DOI: 10.36730/ideologiekritik.2019.4.

Brosda, Carsten. 2008. Diskursiver Journalismus. Journalistisches Handeln zwischen kommunikativer Vernunft und mediensystemischem Zwang. Wiesbaden: VS.

Butterwegge, Christoph, Bettina Lösch, und Ralf Ptak. 2017. Kritik des Neoliberalismus. 3. Auflage. Wiesbaden: Springer VS.

Campbell, Colin. 2018 (1987). The Romantic Ethic and the Spirit of Modern Consumerism. New Extended Edition. Cham: Palgrave Macmillan.

Dietz, Georg. 2018. Klimawandel: Die Katastrophe hätte verhindert werden können. Spiegel Online vom 5.8., http:/ / www.spiegel.de/kultur/gesellschaft/klimawandel-die-katastrophe-haette-verhindert-werden-koennen-a-1221528.html. Zugegriffen: 15. November 2019 .

Dullien, Sebastian. 2016. „Fortgeschrittene Makroökonomie-Lehrbücher mit Tunnelblick. Über die Enge der DSGE-Modellwelt“. In Wirtschaft neu denken. Blinde Flecken der Lehrbuchökonomie, herausgegeben von Till van Treeck und Janina Urban, 200-209. Berlin: iRights Media, http://www.fgw-nrw.de/fileadmin/user_upload/Blinde_Flecken_der_Lehrbuchoekonomie_klein.pdf. Zugegriffen: 15. November 2019. 
Elsner, Wolfram. 2016. „Dichotomien, Inkonsistenz, merkwürdige Antiquiertheit. Mainstream-Mikro-Lehrbücher: Das Beispiel sozialer Institutionen“. In Wirtschaft neu denken. Blinde Flecken der Lehrbuchökonomie, herausgegeben von Till van Treeck und Janina Urban, 42-57. Berlin: iRights Media, http://www.fgw-nrw.de/fileadmin/user_upload/Blinde_Flecken_der_Lehrbuchoekonomie_klein.pdf. Zugegriffen: 15. November 2019.

Ferschli, Benjamin, Daniel Grabner, und Hendrik Theine. 2019. Zur Politischen Ökonomie der Medien in Deutschland. Eine Analyse der Konzentrationstendenzen und Besitzverhältnisse. ISW-Report Nr. 118, München: Institut für sozial-ökologische Wirtschaftsforschung.

Fromm, Erich. 1979. Haben oder Sein. Die seelischen Grundlagen einer neuen Gesellschaft. München: dtv.

Göpel, Maja. 2016. The Great Mindshift. How a New Economic Paradigm and Sustainability Transformations go Hand in Hand. Wuppertal Institut und Springer Open, Open Access: https://www.springer.com/de/book/9783319437651. Zugegriffen: 15. November 2019.

Graupe, Silja. 2016. „Der erstarrte Blick. Eine erkenntnistheoretische Kritik der Standardlehrbücher der Volkswirtschaftslehre". In Wirtschaft neu denken. Blinde Flecken der Lehrbuchökonomie, herausgegeben von Till van Treeck und Janina Urban, 18-29. Berlin: iRights Media, http://www.fgw-nrw.de/fileadmin/user_upload/Blinde_Flecken_der_Lehrbuchoekonomie_klein.pdf. Zugegriffen: 15. November 2019.

Harari, Yuval Noah. 2015. Eine kurze Geschichte der Menschheit. München: Pantheon.

Heise, Arne, und Sebastian Thieme. 2015. „Zur De-Pluralisierung der Wirtschaftswissenschaft nach 1970: Explorationen in einem wissenschaftlichen Macht- und Kampffeld“. Schmollers Jahrbuch $135 \quad$ (2): 155-188, $\underline{\text { https://www.wiso- }}$ net.de/document/SJB_A50A9221B93C8EF59F2E13AB1F72501C. Zugegriffen: 15. November 2019.

Herman, Edward S. und Chomsky, Noam. 2002. Manufacturing Consent. The Political Economy of the Mass Media. New York: Pantheon.

Hochstrasser, Franz. 2013. Konsumismus. Kritik und Perspektiven. München: Oekom.

Jaeggi, Rahel. 2009. „Was ist Ideologiekritik?“. In Was ist Kritik?, herausgegeben von Rahel Jaeggi und Tilo Wesche, 266-295. Frankfurt am Main: Suhrkamp.

Knauß, Ferdinand. 2016. Wachstum über Alles? Wie der Journalismus zum Sprachrohr der Ökonomen wurde. München: Oekom.

Krätke, Michael. 1999. Neoklassik als Weltreligion? In Die Illusion der neuen Freiheit - Realitätsverleugnung durch Wissenschaft, herausgegeben von der Loccumer Initiative Kritischer Wissenschaftlerinnen und Wissenschaftler, 100-144, Hannover: Offizin.

Krüger, Uwe. 2019. Meinungsmacht. Der Einfluss von Eliten auf Leitmedien und Alpha-Journalisten - eine kritische Netzwerkanalyse. 2. Auflage. Köln: Herbert von Halem.

Krüger, Uwe. 2019. „Transformative Kommunikationswissenschaft: Die Universität als Change Agent für sozialen Wandel“. In Medienrealität 2019, herausgegeben von Michael Meyen, https://medienblog.hypotheses.org/5640. Zugegriffen: 15. November 2019. 
Krüger, Uwe und Michael Meyen. 2018. „Auf dem Weg in die Postwachstumsgesellschaft. Plädoyer für eine transformative Kommunikationswissenschaft". Publizistik 63 (3): 341-357, https://www.researchgate.net/publication/325861731_Auf_dem_Weg_in_die_Postwachstumsgesellschaft_Pladoyer_fur_eine_transformative_Kommunikationswissenschaft_Ein_Beitrag_zur_Selbstverstandnisdebatte_im_Forum_Publizistik_Heft_3_2015_Heft_3_und_4_2016_Hef. Zugegriffen: 15. November 2019.

Leiss, William, Stephen Kline, und Sut Jhally. 1986. Social Communication in Advertising: Persons, Products, and Images of Well-Being. Toronto: Methulen.

Lessenich, Stephan. 2016. Neben uns die Sintflut. Die Externalisierungsgesellschaft und ihr Preis. München: Hanser Berlin.

Mankiw, N. Gregory, und Mark P. Taylor. 2018. Grundzüge der Volkswirtschaftslehre. 7., überarbeitete Auflage. Stuttgart: Schäffer-Poeschel.

Marx, Karl. 1971. Das Kapital. Erster Band. Marx Engels Werke (MEW) Band 23. Berlin (Ost): Dietz.

Meadows, Donella H., Dennis L. Meadows, Jørgen Randers, und William W. Behrens. 1972. The Limits to Growth: A Report for the Club of Rome's Project on the Predicament of Mankind. New York: Universe Books. https://www.dartmouth.edu/ library/digital/publishing/meadows/ltg/. Zugegriffen: 15. November 2019.

Meyen, Michael. 2017. Die (doppelt) konservative Wende in der Kommunikationswissenschaft. Vortrag auf der Gründungstagung des Netzwerks Kritische Kommunikationswissenschaft, Ludwig-Maximilians-Universität München 30.11.-1.12.2017, online: https:// www.youtube.com/watch?v=Qtium9RjnpQ\&feature=youtu.be ( $\mathrm{min}$. 0:53:04 bis 1:15:30). Zugegriffen: 15. November 2019.

Mills, Charles Wright. 1956. The Power Elite. Oxford: Oxford University Press.

Petschow, Ulrich, Steffen Lange, David Hofmann, Eugen Pissarskoi, Nils aus dem Moore, Thorben Korfhage, Annekathrin Schoofs, Hermann Ott. 2018. Gesellschaftliches Wohlergehen innerhalb planetarer Grenzen. Der Ansatz einer vorsorgeorientierten Postwachstumsposition. Zwischenbericht des Projektes „Ansätze zur Ressourcenschonung im Kontext von Postwachstumskonzepten". Dessau-Roßlau: Umweltbundesamt, https:// www.umweltbundesamt.de/sites/default/files/medien/1410/publikationen/uba_texte_89_2018_vorsorgeorientierte_postwachstumsposition.pdf. Zugegriffen: 15. November 2019.

Polanyi, Karl. 1957. The Great Transformation: The Political and Economic Origins of Our Time. Boston, MA: Beacon Press.

Rebhan, Christian. 2017. Einseitig oder plural? Eine quantitative Analyse der wirtschaftswissenschaftlichen Einführungslehrbücher an deutschen Hochschulen. Marburg: Metropolis.

Rosa, Hartmut. 2016. Resonanz. Eine Soziologie der Weltbeziehung. Frankfurt am Main: Suhrkamp.

Scheu, Andreas M. 2012. Adornos Erben in der Kommunikationswissenschaft. Eine Verdrängungsgeschichte? Köln: Herbert von Halem.

Schmuck, Peter. 2015. Die Kraft der Vision. Plädoyer für eine neue Denk- und Lebenskultur. München: Oekom. 
Scholl, Armin. 2016. „Journalismustheorie und Methodologie“. In Handbuch Journalismustheorien, herausgegeben von Martin Löffelholz und Liane Rothenberger, 91-110. Wiesbaden: Springer VS.

Sevignani, Sebastian. 2016. Privacy and Capitalism in the Age of Social Media. New York: Routledge.

Steffen, Will, Wendy Broadgate, Lisa Deutsch, Owen Gaffney, und Cornelia Ludwig. 2015. „The Trajectory of the Anthropocene: The Great Acceleration“. The Anthropocene Review 2 (1): 81-98, doi: https:// doi.org/10.1177/2053019614564785

van Treeck, Till. 2016. „Der ,große Zwiespalt' zwischen Effizienz und Gerechtigkeit: Realität oder Ideologie?" In Wirtschaft neu denken. Blinde Flecken der Lehrbuchökonomie, herausgegeben von Till van Treeck und Janina Urban, 128-139. Berlin: iRights Media, http://www.fgw-nrw.de/fileadmin/user_upload/Blinde_Flecken_der_Lehrbuchoekonomie_klein.pdf. Zugegriffen: 15. November 2019.

van Treeck, Till, und Urban, Janina. 2016. „Einleitung“. In Wirtschaft neu denken. Blinde Flecken der Lehrbuchökonomie, herausgegeben von Till van Treeck und Janina Urban, 6-16. Berlin: iRights Media, http://www.fgw-nrw.de/fileadmin/user_upload/Blinde_Flecken_der_Lehrbuchoekonomie_klein.pdf. Zugegriffen: 15. November 2019.

WBGU (Wissenschaftlicher Beirat der Bundesregierung Globale Umweltveränderungen). 2011. Welt im Wandel. Gesellschaftsvertrag für eine Große Transformation. Bonn. https:// www.wbgu.de/fileadmin/user_upload/wbgu/publikationen/hauptgutachten/hg2011/pdf/wbgu_jg2011.pdf. Zugegriffen: 15. November 2019.

Welzer, Harald. 2013. Selbst denken. Eine Anleitung zum Widerstand. Frankfurt am Main: S. Fischer.

Williamson, Judith. 2000. Decoding advertisements: Ideology and meaning in advertising. London: Marion Boyars.

\section{Open Access}

Dieser Beitrag erscheint unter der Creative-Commons-Lizenz CC-BY-SA 4.0: https://creativecommons.org/licenses/by-sa/4.0/ 